\title{
Análisis de la adquisición de vivienda a través de créditos hipotecarios con instrumentos de prorrateo de edad
}

\author{
L. Enrique Aranda-Guerrero, MVii \\ J. Manuel Lozano-de Poo, PhD \\ Universidad Autónoma de San Luis Potosí, México
}

Doi:10.19044/esj.2022.v18n1p86

Submitted: 29 October 2021

Accepted: 18 January 2022

Published: 31 January 2022
Copyright 2022 Author(s)

Under Creative Commons BY-NC-ND

4.0 OPEN ACCESS

Cite As:

Aranda-Guerrero L.E. \& Lozano-de Poo J.M. (2022). Análisis de la adquisición de vivienda a través de créditos hipotecarios con instrumentos de prorrateo de edad. European Scientific Journal, ESJ, 18 (1), 86. https://doi.org/10.19044/esj.2022.v18n1p86

\section{Resumen}

Identificar áreas en la zona metropolitana de San Luis Potosí (ZMSLP) para la compra de vivienda usada susceptible a un crédito hipotecario, genera que los personas no tengan que adquirir alguna propiedad en zonas retiradas de su espacio de movimiento. El objetivo de este trabajo es detectar las áreas donde se han recuperado distintos tipos de edificaciones para uso habitacional por medio de un análisis de las valuaciones realizadas en los últimos meses, determinando áreas de oportunidad para la adquisición de viviendas susceptibles a reincorporarse al mercado inmobiliario. Este diagnóstico refleja la oportunidad de evaluar la posibilidad de comprar algún patrimonio mediante un producto bancario o crédito gubernamental. Estos proyectos reciben una intervención física que ayuda a disminuir su vida útil normal, mejorando sus condiciones de habitabilidad. Para esta investigación se revisaron 119 propiedades en un período de 6 meses, de las cuales solamente 35 presentaron la opción de aplicación de la tabla de prorrateo de edad con distintas características físicas y de ubicación. Esta tabla es un instrumento que se utiliza en los procesos de valuación profesional para mejorar los porcentajes de trabajo en las partidas de construcción. Los resultados de esta investigación establecen las áreas habitacionales donde se han llevado a cabo dichas intervenciones e indica las colonias donde se pueden remodelar o adecuar espacios para un segundo tiempo de uso, así como las características generales que presentan; sirviendo como vitrina para evaluar y comparar dentro de la toma de decisiones. 
Palabras claves: Edad prorrateada, Valor de Reposición Nuevo, Mercado Abierto Individual, Vida Útil Normal

\title{
Analysis of Home Purchase Through Mortgage Loans with Age Apportionment Instruments
}

\author{
L. Enrique Aranda-Guerrero, MVii \\ J. Manuel Lozano-de Poo, PhD \\ Universidad Autónoma de San Luis Potosí, México
}

\begin{abstract}
Identifying areas in the metropolitan zone of San Luis Potosí (ZMSLP) for the purchase of used housing susceptible to a mortgage loan, means that people do not have to acquire a property in remote areas of their space of movement. The objective of this work is to detect the areas where different types of buildings for residential use have been recovered through an analysis of the valuations carried out in recent months, determining areas of opportunity for the acquisition of homes susceptible to rejoining the real estate market. This diagnosis reflects the opportunity to evaluate the possibility of buying a patrimony through a bank product or government credit. These projects receive a physical intervention that helps reduce their normal useful life, improving their living conditions. For this research, 119 properties were reviewed in a 6-month period, of which only 35 presented the option of applying the age apportionment table with different physical and location characteristics. This table is an instrument used in professional valuation processes to improve work percentages in construction items. The results of this research establish the residential areas where these interventions have been carried out and indicate the neighborhoods where spaces can be remodeled or adapted for a second time of use, as well as the general characteristics they present; serving as a showcase to evaluate and compare within decision making.
\end{abstract}

Keywords: Prorated Age, New Replacement Value, Individual Open Market, Normal Useful Life

\section{Introduction}

Según datos del sector de la construcción de vivienda, este ramo del desarrollo económico contribuye con un $6 \%$ del PIB en México. Los desarrollos habitacionales en el país contribuyen de manera importante en el movimiento económico, detonan otros sectores mercantiles que generan 
ingreso y bienestar en la población. Por lo que la compra y venta de vivienda nueva o usada forman parte de una estructura dinámica para el ejercicio de otras instituciones públicas y privadas.

El Subdirector Gerente del Fondo Monetario Internacional (FMI) comento en una de sus conferencias, "El sector inmobiliario es esencial para la economía, pero también es un sector que ha sido fuente de vulnerabilidad y crisis" (Zhu, 2014) en este sentido podemos visualizar la importancia del campo inmobiliario y sus efectos en la economía de cada país.

Los escenarios económicos actuales se tornan confusos. Los efectos de la pandemia y la baja en los precios del petróleo han golpeado severamente al gremio de la construcción. Las empresas dedicadas a la edificación de proyectos habitacionales han tenido que recurrir a distintos instrumentos financieros para lograr continuar con metas y objetivos. Las instituciones de otorgamiento de crédito Sociedad Hipotecaria Federal (SHF, 2001) han apoyado para proporcionar fondos para la gestión de créditos hipotecarios de los bancos y otras dependencias diversas. Evitar los descalabros como en 1994 y 2008 donde el sector de la construcción fue afectado por el exceso en la aprobación de créditos para la adquisición de vivienda, por una falta de organismos de control y regulación, han servido como experiencia para prevenir situaciones problemáticas.

El autor Fidel Álvarez González comenta en su libro "Introducción a la Valuación de Inmuebles" en su capítulo VI de economía, escribe que, en esta se deben de establecer como se producen los distintos productos y servicios, así como determinar los diferentes mecanismos que los generan, para finalmente especificar quiénes son las personas o grupos que necesita $\mathrm{o}$ requieren tales insumos, por lo tanto la actividad económica es medular para continuar con el desarrollo y crecimiento, el mercado inmobiliario participa de forma trascendental, detonando operaciones mercantiles para los habitantes de cualquier sector social. (Álvarez, 2012, pág. 146)

El sector inmobiliario cada día toma mayor fuerza y se presenta más competitivo. Año tras año, surgen nuevas empresas constructoras con mejores estrategias de mercado y dominio en el manejo de los créditos hipotecarios. Y precisamente los procesos de otorgamiento de créditos son un tema fundamental para los jóvenes, quienes actualmente analizan la posibilidad de obtener alguno.

Como se menciona en el libro de "Phoenix, Valuación Inmobiliaria" referente al tema del mercado inmobiliario, en él se describe que "En el intervienen un conjunto de compradores potenciales con la necesidad de satisfacer la adquisición, venta o disfrute de un bien inmueble" (Cordova, 2017, pág. 10) siendo así un menester la compra de un bien inmueble en un momento determinado, por alguna persona, con el objetivo de poseer un patrimonio, 
tarde o temprano las personas estudian la posibilidad de adquirir un espacio propio para vivir.

La actividad valuatoria evoluciona cada día, nuevos sistemas, aplicaciones se ofrecen para mejorar el ejercicio y la práctica. El compromiso del valuador representa retos nuevos, actualización constante para ser pieza clave en el desarrollo económico de su localidad.

"No obstante la alta responsabilidad del profesional de la valuación, del desarrollo de las tecnologías de la información y el incremento del tamaño del mercado, se observa en particular en el rubro de elementos tecnológicos" (Pérez, 2007)

En la actualidad los jóvenes que estudian nivel superior requieren conocer el comportamiento del mercado de vivienda, su oferta y demanda. El alumno universitario visualiza en un futuro adquirir un espacio propio, conformar un patrimonio y poseer así un lugar para vivir cómodamente. En este sentido, el sector inmobiliario ofrece una gran diversidad de opciones para comprar un bien inmueble para fines domésticos; desde casas de interés social, hasta de tipo residencial.

Por lo tanto, es vital que los jóvenes identifiquen los puntos fundamentales en el proceso de gestión de un crédito hipotecario. Debido a que, posteriormente al inicio de su vida laboral, recibirán por parte del patrón la prestación de seguro social, misma que les otorgará el beneficio de solicitar un crédito y adquirir una vivienda en corto plazo (ver imagen 1). Lo anterior obedece a que generalmente existe desconocimiento por parte de los jóvenes sobre las opciones y oportunidades que ofrece el Instituto del Fondo Nacional de la Vivienda para los Trabajadores (INFONAVIT), institución que ofrece una variedad de oportunidades para la compra de un patrimonio.

En el libro "Valoración Inmobiliaria, Métodos y Aplicaciones" define a los bienes inmuebles como terreno y cualquier otra cosa que sea parte de los mismos, refiriéndose a los distintos elementos que el hombre pueda integrar e incorporar a un predio, como por ejemplo edificios o mejoras (Bellver, 2021, pág. 23). Esto nos permite contextualizar e identificar el espacio donde se desarrollar el movimiento mobiliario, sus alcances y razones, permite reflexionar sobre el comportamiento económico que se presenta actualmente, sus causas y efectos, así como la necesidad natural del ser humano de poseer un hogar. Otros autores utilizan el termino propiedad "La valoración inmobiliaria trata de discernir el valor de una determinada propiedad, constituida generalmente por suelo, edificación o inmueble" (Garcia, 2007, pág. 8) por lo tanto se entiende que los bienes inmuebles están relacionados con los elementos de terreno o pedio con las edificaciones que se realicen en su interior. 


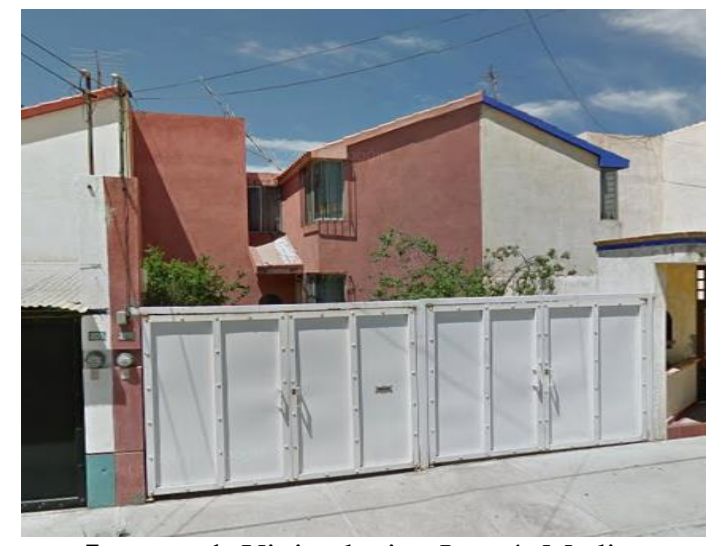

Imagen 1. Vivienda tipo Interés Medio. Elaboración propia. 2021

El campo de la valuación de bienes inmuebles evoluciona constantemente y requiere de actualizar sus mecanismos para su operatividad. El ejercicio de nuevos créditos para la adquisición de vivienda es una actividad constante por los agentes inmobiliarios que promueven la compraventa de patrimonio para los jóvenes que pueden acceder a créditos tradicionales como: INFONAVIT, Bancarios y del Fondo de la Vivienda de Seguridad y de Servicios Sociales de los Trabajadores del Estado (FOVISSSTE). De esta manera, el crecimiento de la población es un indicador importante que genera la exigencia en la adquisición de vivienda y por lo tanto, representa un incremento del número de solicitudes de créditos hipotecarios, tanto para viviendas nuevas, como para viviendas usadas Mercado Abierto (MAI).

El proceso para acceder a esto créditos se puede sintetizar en los siguientes pasos. i) Se estudian y analizan los posibles compradores a través de su historial crediticio, liquidez, registro en el buró de crédito, así como las posibilidades reales que tienen para recibir un crédito. ii) Se diseñan créditos hipotecarios según los ingresos y su capacidad de pago. Cabe mencionar que las diferentes instituciones bancarias promueven sus productos hipotecarios, haciendo énfasis principalmente en las bondades de su tasa de interés. iii) Posteriormente el cliente debe evaluar otros aspectos como: las comisiones de apertura de crédito, gastos notariales, costo del avalúo, seguro del crédito y las características más específicas del crédito (pronto pago, adelantos de mensualidades y plazo del crédito).

Las instituciones financieras cuentan con un departamento de créditos hipotecarios, los cuales establecen metas y estrategias cada año para colocar sus créditos. Estas áreas tienen el objetivo de otorgar la mayor cantidad de créditos, tanto para construcción de vivienda como para la adquisición individual de bienes inmuebles. Por lo general, los tiempos de duración de un crédito hipotecario pueden ser de 10, 15 y hasta 20 años. 
En el mercado de vivienda, existen dos opciones para el financiamiento por parte de las instituciones anteriormente mencionadas. El primero es la vivienda nueva o de paquete y el segundo es la vivienda usada MAI "Opción que tienen los acreditados para ejercer su crédito para la adquisición de vivienda nueva o usada en forma individual, no en paquete" (Mello, 2007) Para este caso analizaremos el tema de la vivienda usada o de mercado abierto. Es indispensable aclarar que, para obtener un crédito hipotecario para la adquisición de una vivienda usada, el inmueble debe contar con una edad menor a 30 años, ya que la vida útil normal de una vivienda de interés social es de 60 años. En caso de superar los 30 años no es factible asignarle un crédito hipotecario debido a que la vida remanente no alcanzaría a cubrir el tiempo del crédito, "la vida útil normal se define como el período, expresado en años en el cual puede esperarse razonablemente que un bien realice la función para la cual fue construido, a partir de la fecha en que fue puesto en servicio" (Córdova, 2019, pág. 252).

\section{Zonas de Vivienda usada}

En la capital del estado de San Luis Potosí se localizan diversas zonas de vivienda usada que superan los 30 años. El mercado de clientes, para la compra de este tipo de edificaciones es constante aún y cuando presenten edad avanzada, derivado de que cuentan con buena ubicación, servicios e infraestructura, a diferencia de las colonias de la periferia de la Zona Metropolitana de San Luis Potosí (ZMSLP). De esta manera, las viviendas que se construyeron en fraccionamientos en las décadas de 1970 y 1980 son una opción viable de compra por parte del sector joven de la población.

En este sentido, las unidades de valuación son las entidades encargadas de realizar y vigilar las valuaciones a los inmuebles que son susceptibles a obtener un crédito hipotecario. Estas unidades son auditadas constantemente por la SHF. Anteriormente, al momento de presentarse la solicitud de un crédito para valuar un inmueble que rebasara los 30 años, ésta era automáticamente rechazada. Para resolver esta situación las unidades de valuación han diseñado instrumentos para prorratear su edad que-enlistan las partidas de obra que presenta una vivienda, cada una tiene un porcentaje según su costo de participación en el proyecto. "El surgimiento de las Unidades de Valuación en México se debe al mandato establecido por el Congreso en fecha 3 de abril de 2001" (Guerrero, Hernández, \& Pacheco, 2007, pág. 28)

Cuando en la vivienda se comprueba que ha recibido trabajos de mejoras o de remodelación, estos porcentajes son determinados nuevamente. Lo anterior, conlleva a una disminución de su edad y aumento en su vida remanente, dando como resultado que el crédito hipotecario sea factible.

La implementación de estos nuevos instrumentos ha sido de gran beneficio, ya que rescata una importante cantidad de viviendas que todavía 
pueden ser viables para ser habitadas y canalizadas para un crédito hipotecario (ver imagen 2). La ZMSLP cuenta con este segmento de inmuebles que generan y reactivan el desarrollo económico y como consecuencia estas colonias se pueden volver a habitar, así como el efecto financiero que producen con las instituciones bancarias, unidades de valuación, valuadores, controladores, brokers y agentes inmobiliarios. Estos mecanismos ayudan a que los jóvenes puedan adquirir una vivienda en una zona más accesible a su área de trabajo y no tengan que emigrar a fraccionamientos ubicados en la periferia de la ciudad.

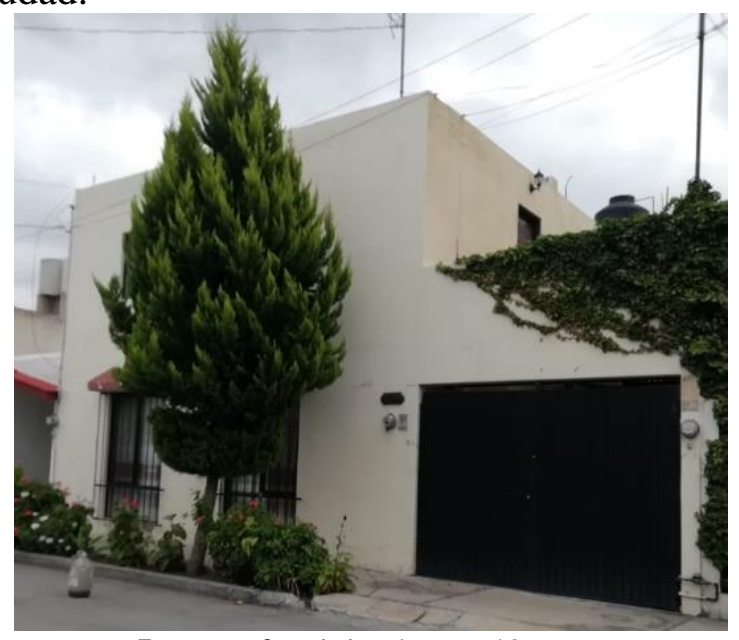

Imagen 2. Vivienda con 40 años.

Elaboración propia. 2021

La estructura de la tabla de prorrateo está conformada por una hoja cálculo de Excel. En la primera columna se enlistan las distintas partidas que componen un presupuesto de obra. En la segunda columna se muestran los porcentajes de incidencia por cada partida. En la tercera columna se muestra el resultado de multiplicar el Valor de Reposición Nuevo (VRN) del inmueble analizado por el porcentaje de incidencia. En la cuarta columna se establecen los porcentajes de avance de las partidas que han sido intervenidas, estos porcentajes son vaciados por el valuador al realizar la visita de inspección al inmueble. En la última columna se coloca el resultado de multiplicar el porcentaje de incidencia (segunda columna) por el porcentaje de trabajo de remodelación (cuarta columna) dando como resultado el porcentaje mejorado en cada partida. Finalmente se realiza la sumatoria de esta lista de porcentajes que determinan el total de trabajo de mejora de este inmueble, se hace una conversión en años para así obtener le nueva edad del inmueble (ver Imagen $3)$. 


\begin{tabular}{|c|c|c|c|c|}
\hline $\begin{array}{l}\text { VRN DEL INMUEBLE EN } \\
\text { CONDICIONES NORMALES (A) }\end{array}$ & $\$ 6,200.00$ & \multicolumn{3}{|c|}{ FUENTE: MANUAL DE COSTOS UNITARIOS BIMSA } \\
\hline EDAD REAL DEL INMUEBLE & 53 & \multicolumn{2}{|c|}{$\begin{array}{l}\text { EDAD DE LA REMODELACION } \\
\text { (TIEMPO TAANSCURRIDO DESDE LA REMODELACION) }\end{array}$} & 5 \\
\hline PARTIDAS & $\%$ & PRECIO & REMODELACION & $\begin{array}{l}\text { PORCENTAIE } \\
\text { APICABE }\end{array}$ \\
\hline CIMENTACIÓN & $12.99 \%$ & 5805.38 & $0.00 \%$ & $0.00 \%$ \\
\hline ESTRUCTURA DE CONCRETO & $19.54 \%$ & $51,211.48$ & $0.00 \%$ & $0.00 \%$ \\
\hline ALBAÑIILERIA & $10.41 \%$ & 5645.42 & $70.00 \%$ & $7.29 \%$ \\
\hline INSTALACIOON HIDRÁULICA & $2.98 \%$ & $\frac{5184.76}{5989}$ & $70.00 \%$ & $2.09 \%$ \\
\hline \begin{tabular}{|l} 
INSTALACIÓN SANITARIA \\
IIISAAACOON EEETCRIIA
\end{tabular} & $\frac{1.44 \%}{0.09 \%}$ & 589.28 & $\frac{70.00 \%}{7000 \%}$ & $1.01 \%$ \\
\hline INSTALACIÓN DE GAS & $0.15 \%$ & 59.30 & $70.00 \%$ & $0.62 \%$ \\
\hline ACABADOS INTERIORES & $14.85 \%$ & 5920.70 & $90.00 \%$ & $13.37 \%$ \\
\hline ACABADOS EXTERIORES & $11.74 \%$ & 5727.88 & $90.00 \%$ & $10.57 \%$ \\
\hline ACCESOS & $1.96 \%$ & $\$ 121.52$ & $100.00 \%$ & $1.96 \%$ \\
\hline CAnCELERIA & $2.55 \%$ & 5158.10 & $100.00 \%$ & $2.55 \%$ \\
\hline MOBILARIO FIJO & $9.60 \%$ & $\$ 595.20$ & $100.00 \%$ & $9.60 \%$ \\
\hline EQUIPO & $0.25 \%$ & 515.50 & $100.00 \%$ & $0.25 \%$ \\
\hline \multirow{3}{*}{$\begin{array}{l}\text { GUARUA } \\
\text { ACCESORIAS } \\
\end{array}$} & $\frac{3.81 \%}{6.84 \%}$ & 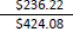 & $\frac{100.00 \%}{10000 \%}$ & $3.81 \%$ \\
\hline & $100.00 \%$ & $\$ 6,200.00$ & PORCENTAE RESTANTE DE NO REMODELACION & $40 \%$ \\
\hline & & & PORCENTAUE DE REMODELACION & $60 \%$ \\
\hline \multirow{3}{*}{$\begin{array}{c}\text { Vida probable } \\
\text { tesoreria }\end{array}$} & \multirow{3}{*}{70} & & Área sin remodelar & $39.95 \%$ \\
\hline & & & Edad sin remodelar & 53 \\
\hline & & & Area remodelada & $60.05 \%$ \\
\hline & & & Edad remodelada & 5.00 \\
\hline & & & EDAD PRORRATEADA & 24.18 \\
\hline
\end{tabular}

Imagen 3. Tabla de prorrateo de edad. CTI.

Consorcio Técnicos Inmobiliarios S.A. de C.V., 2021.

Para contextualizar respecto a el significado del término valor de reposición nuevo se analizó la definición de Costo de Reposición Nuevo, que menciona: "es el costo actual de un bien valuado, considerándolo como nuevo, con sus gastos de ingeniería y de instalación, en condiciones de operación, precio de contado" (Córdova, 2019, pág. 322) por lo tanto se debe de proponer un valor que contemple todos los trabajos necesarios para edificar un bien con el mismo sistema constructivo, acabados, alturas y especificaciones marcadas. Por otro lado, en el Diario Oficial de la Federación menciona como "costo a precios actuales de un inmueble nuevo similar, que tenga la utilidad o la función equivalente más próxima al inmueble objeto de estudio" (Diario Oficial, 2004, pág. 2)

En el libro "Costo y Tiempo en Edificación" menciona que el análisis de este es aproximado, ya que, al no existir dos procesos constructivos iguales, el intervenir la habilidad personal del operario, y el basarse en condiciones promedio de consumos, insumos y desperdicios (Salazar, 2013, pág. 22) por lo tanto para establecer el costo de un tipo de construcción, es necesario realizar un análisis del sistema constructivo, materiales, acabados y especificaciones propias del proyecto.

\section{Metodología}

En México, el sector de la valuación inmobiliaria es regulado por la Secretaría de Economía, institución federal que vigila los procesos en este sector, tal como lo menciona José Manuel Salas Tafoya en "El Modelo de Valuación Inmobiliaria en México", explicando que la norma NMX-C-459- 
SCFI-ONNCCE-2007 se enumeran los lineamientos para desarrollar esta actividad. Es importante realizar con calidad profesional y ética el servicio de valuar bienes inmuebles, y se identifica en el segundo paso, en donde determina la eficiencia en este ejercicio: La inspección de los bienes a valuar para constatar su existencia identificación y ubicación (Salas, 2015). Por consiguiente, podemos establecer que existen distintas metodologías implementadas en fases de valuación, tanto por organismos de gobierno como privados.

Se realizó un sondeo, donde se obtuvo información del sistema digital SAX de avalúos de la unidad de valuación Consorcio Técnicos Inmobiliarios, S.A. de C.V. (CTI). Las unidades de valuación son empresas o sociedades mercantiles registradas debidamente ante la SHF, con el objetivo de elaborar avalúos certificados para créditos hipotecarios, respetando la normatividad que se establece y marca este ejercicio, en caso de no realizar correctamente sus actividades son sancionadas severamente con multas o suspensión por periodos de tiempo. Las unidades de valuación en nuestro país tienen como antecedente la circular 1462 de la Comisión Nacional Bancaria y de Valores (CNBV) (Guerrero, Hernández, \& Pacheco, 2007, pág. 24) Se tomaron datos a partir del mes de octubre de 2020, hasta el mes de marzo de 2021 de 119 viviendas en el interior del estado y municipio de San Luis Potosí. A continuación, se describen los 5 pasos que se utilizaron para el diagnóstico de los inmuebles.

Paso 1. Se ingresó al sistema SAX para obtener información, en la bandeja de entrada es el espacio donde se enlistan los folios de las solicitudes de avalúos certificados.

Paso 2. Se analizaron los folios partir del día 1 de octubre de 2020 , hasta el último folio del mes de marzo de 2021, es decir un tiempo total de 6 meses, se revisaron cada una de las solicitudes en donde se obtuvo la información relevante, siendo principalmente la ubicación y si utilizo la tabla de prorrateo.

Paso 3. La información obtenida se vació a una hoja de cálculo del programa Excel, donde se separó por medio de columnas, catalogando así la información generada por cada solicitud de avalúo: ubicación, valor del inmueble, institución que otorgó el crédito, por mencionar algunas.

Paso 4. Posteriormente, se graficó solamente la información relevante, esto con el objetivo por obtener datos relacionados principalmente sobre las áreas donde se han aplicado este tipo de instrumentos.

Paso 5. Se realizó la reflexión y depuración de datos, esto con el objeto de elaborar el diagnóstico y evaluación de los resultados de este ejercicio.

Las conclusiones obtenidas en esta investigación se eligieron, de tal forma que se eliminó del proceso, aspectos que no presentaban relación con el objetivo de trabajo. La aportación es lograr identificar las zonas donde se están 
reutilizando estos espacios habitacionales y así poder dar difusión para su aprovechamiento.

\section{Resultados}

El mayor porcentaje de viviendas se concentra en los municipios de San Luis Potosí y Soledad de Graciano Sánchez. Del total de 119 viviendas, solamente 35 avalúos utilizaron esta tabla. En este período de los 35 avalúos analizados, el $93.2 \%$ fueron aprobados para su seguimiento en el proceso del crédito, solamente el $2.5 \%$ fueron cancelados, otro $2.5 \%$ rechazados y finalmente un $1.8 \%$ quedó con estatus de captura (ver gráfica 1). Esta información nos indica que un mayor porcentaje de avalúos son los que terminan correctamente su proceso de elaboración y continúan en el procedimiento de gestión para un crédito hipotecario, lo cual demuestra una correcta y adecuada revisión de documentación previa de los inmuebles.

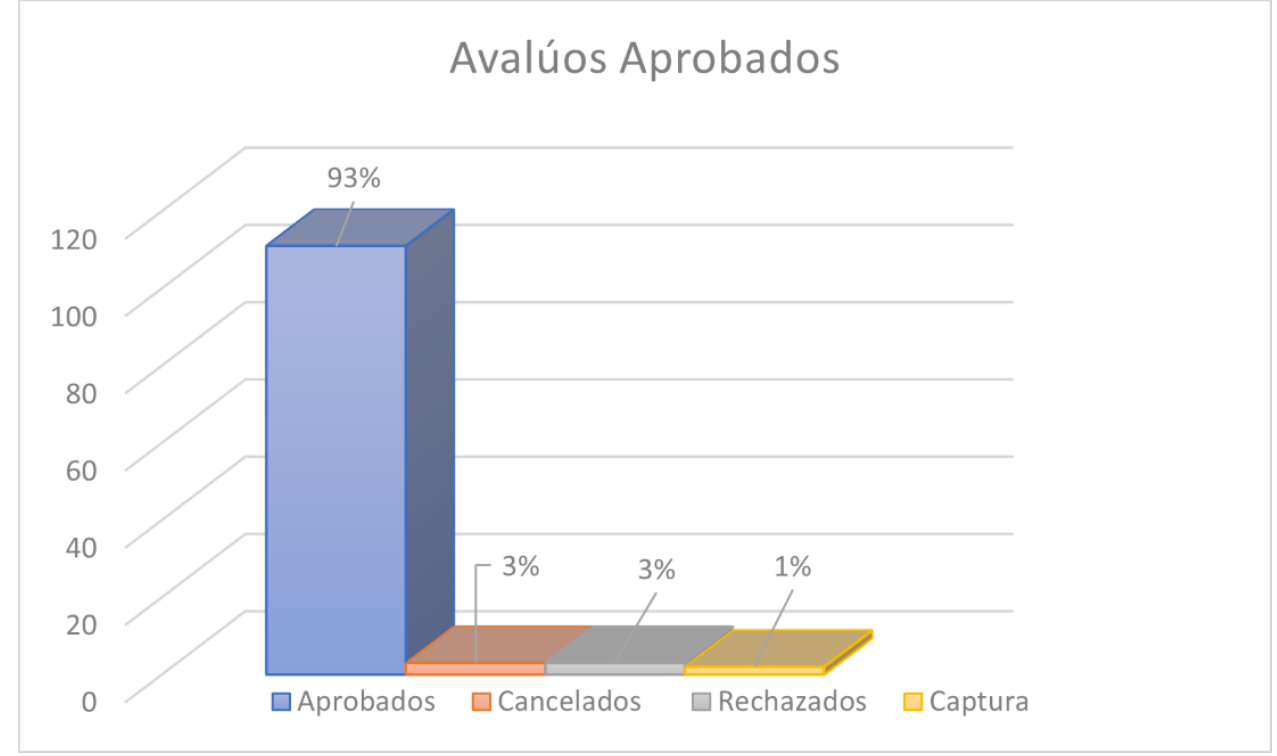

Gráfica 1. Porcentaje de avalúos aprobados. Elaboración propia, 2021

El estado de San Luis Potosí se localiza al Noreste de la República Mexicana, colinda con los estados de: Querétaro, Guanajuato, Hidalgo, Veracruz, Tamaulipas, Nuevo León y Zacatecas. La posición del estado es estratégica, la carretera federal 57 México-Piedras Negras, atraviesa el estado, generando desarrollo económico en la región. La zona industrial del municipio de San Luis Potosí, actualmente está conurbada con el municipio de Villa de Reyes, creando así desde hace varios años un "cluster" en la producción de autopartes para vehículos (ver mapa 1). 


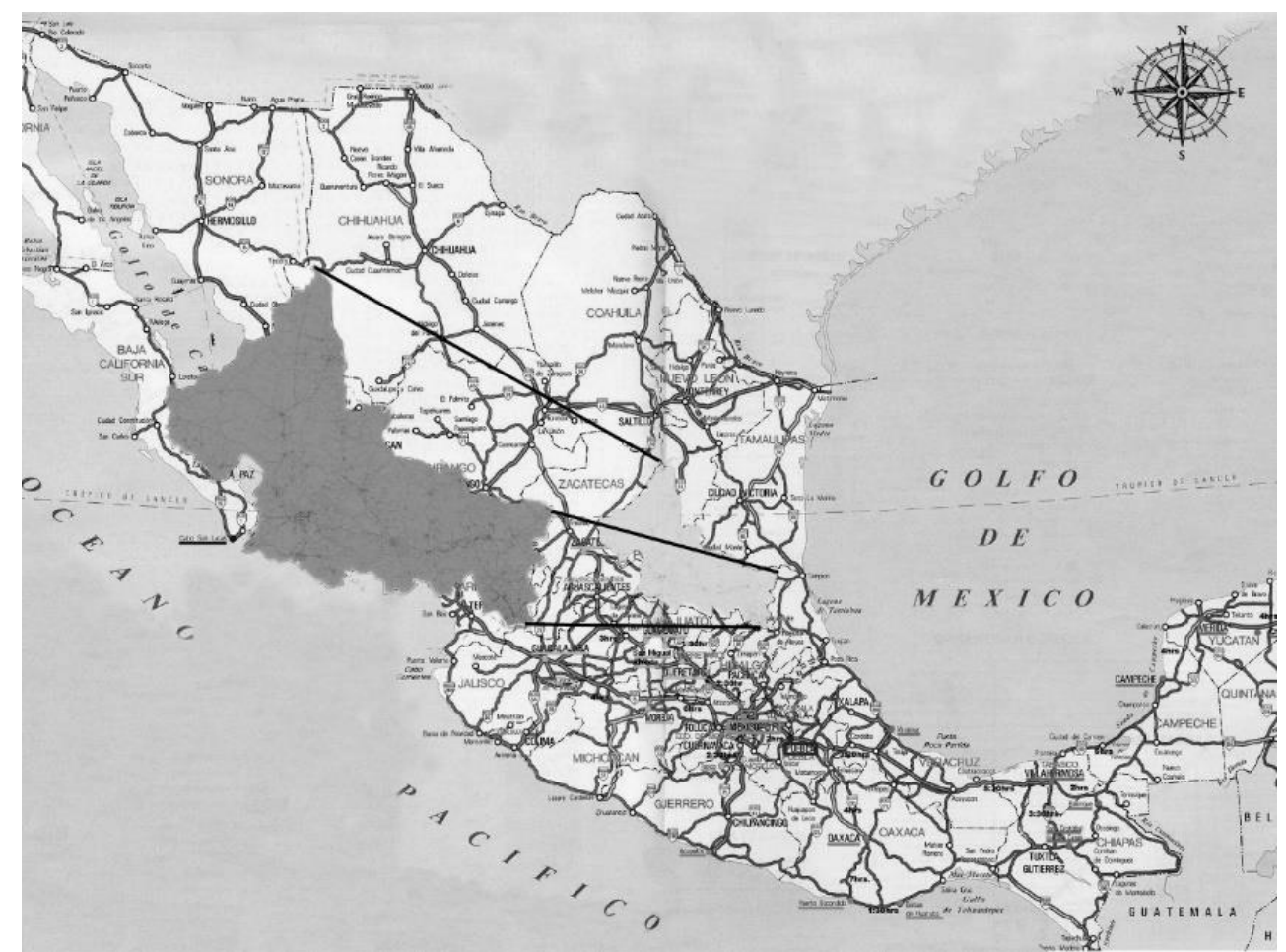

Mapa 1. Mapa República Mexicana indicando ubicación del Estado de San Luis Potosí. Elaboración propia. 2021

El estado de San Luis Potosí está conformado por 58 municipios. Así mismo el estado está dividido en cuatro zonas: centro, altiplano, media y huasteca. El municipio de San Luis Potosí se ubica al suroeste del estado, así mismo el municipio de San Luis Potosí pertenece a la zona centro, colinda con los municipios de: Villa de Reyes, Villa de Zaragoza, Soledad de Graciano Sánchez, Cerro de San Pedro, Villa Hidalgo, Villa de Arista, Moctezuma, Ahualulco, Villa de Arriaga y Mexquitic de Carmona. El municipio de San Luis Potosí está integrado por cuatro delegaciones: Villa de Pozos, La Pila, Bocas y Escalerillas (ver mapa 2). 


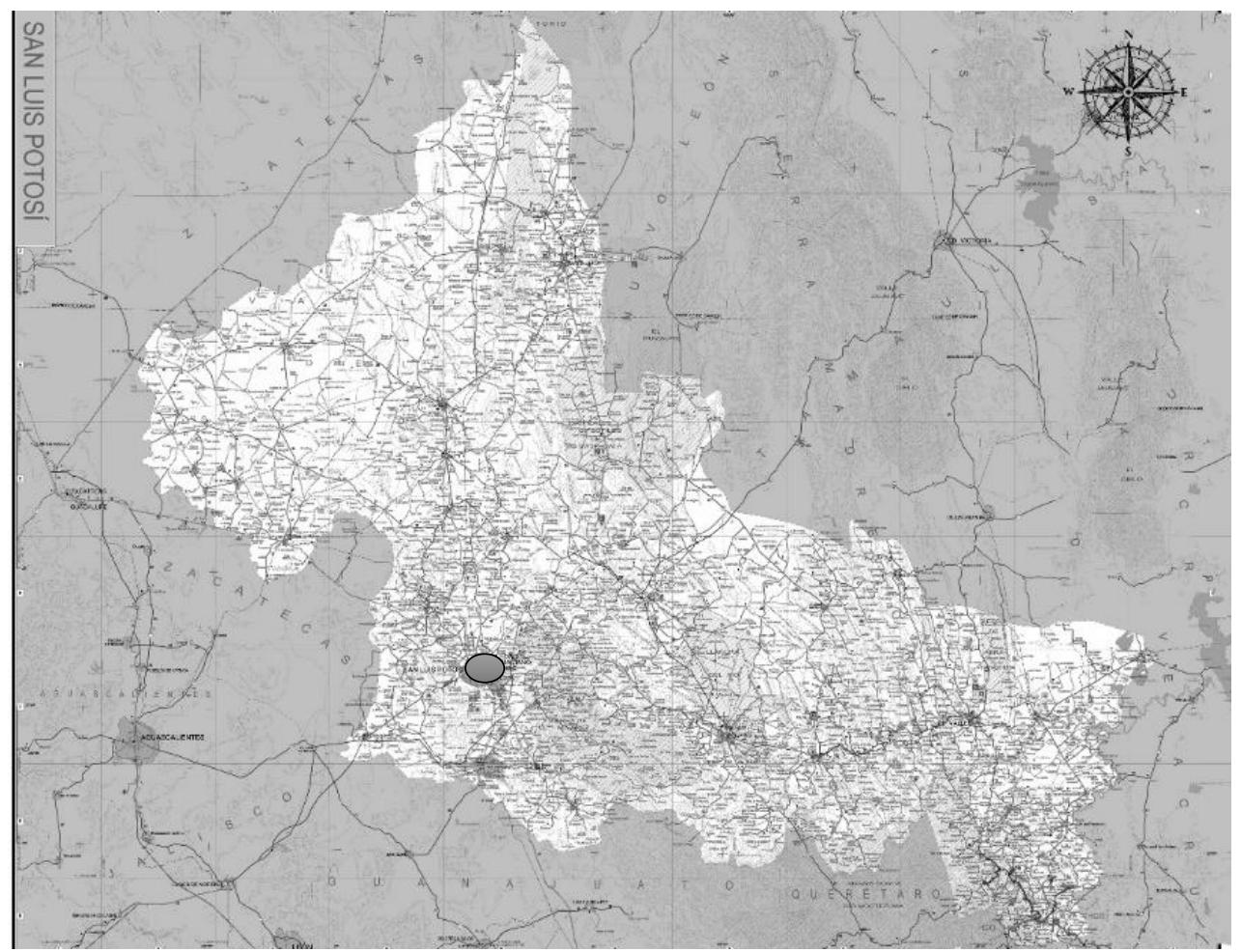

Mapa 2. Mapa del Estado de San Luis Potosí ubicando la mancha urbana del municipio de San Luís Potosí.

Elaboración propia. 2021

El mapa 3 muestra la distribución espacial de los 35 avalúos que utilizaron la tabla de prorrateo de edad Esta información determina que solamente un $29 \%$ del total de valuaciones de vivienda en un período de 6 meses utilizaron este instrumento. El resto de las viviendas presentan una edad menor en donde no es necesario recurrir a este tipo de instrumento para disminuir la edad de los inmuebles y así poder ser susceptibles a un crédito hipotecario. Por lo tanto, la mayor parte de los bienes inmuebles que se gestionan para un crédito son casas habitación o departamentos nuevos o que presentan mejores condiciones, en donde no es necesario realizar una intervención o remodelación física. La mayor parte de estas viviendas se ubican en zonas o fraccionamientos de la periferia y en un segundo plano en zonas intermedias del área metropolitana de los municipios de San Luis Potosí y Soledad de Graciano Sánchez. En la zona suroriente se puede detectar una mayor concentración. 


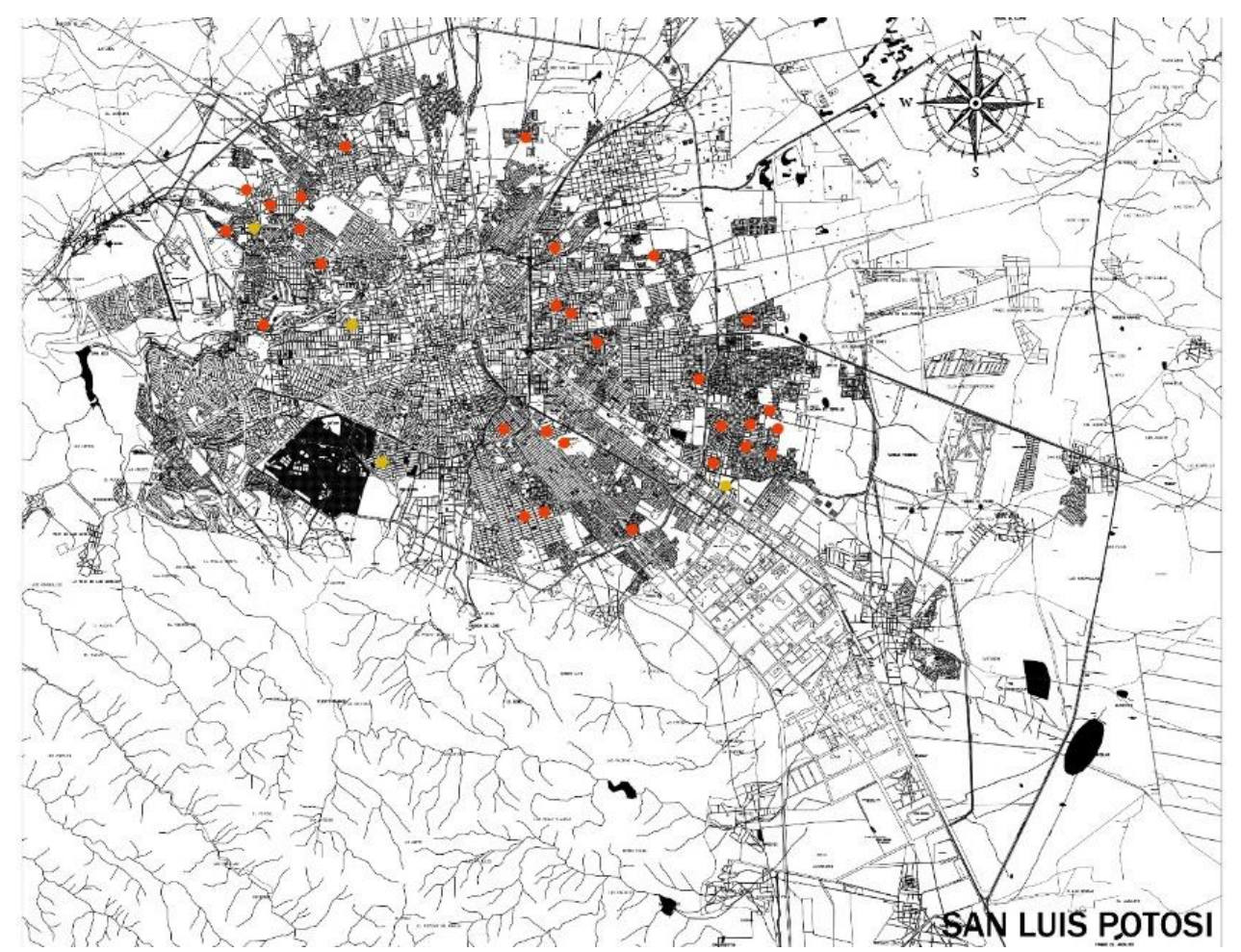

Mapa 3. Ubicación de viviendas donde se aplicó la tabla de prorrateo. Elaboración propia. 2021

Vivienda de interés social Vivienda de interés medio

La Unidad Habitacional Rancho Viejo II, localizada en el municipio de Soledad de Graciano Sánchez, se identifica como la zona donde se ubican una mayor cantidad de departamentos en donde se utilizó la tabla. Estos inmuebles fueron remodelados en su interior para poder disminuir su edad. Generalmente los trabajos que se realizan son: cambio de piso y muebles de baño, suministro y colocación de pintura, puertas y clósets de madera. Solamente en casos especiales se requiere de cambiar totalmente las instalaciones hidráulicas de tubo galvanizado o cable eléctrico, ya que la vida útil de estos materiales ha transcurrido y deben ser remplazados. También hay algunas viviendas que fueron en un tiempo invadidas y se tiene que desarrollar un trabajo de rehabilitación más especializado a elementos de la propia estructura del inmueble, como muros y losas.

En un principio se pensaba que la tabla solamente se aplicaba para viviendas que presentan edad mayor a 30 años, pero al realizar el sondeo se detectó que hay casas habitación o departamentos que tiene menos de 30 años. Esta situación deriva de que se pueden ofrecer en el mercado inmuebles que tengan que recibir algún trabajo previo de restauración a su ofrecimiento en el 
mercado o que simplemente el vendedor toma la decisión de hacer una intervención de remodelación en el inmueble para que así presente una mejor imagen y sea más atractivo para su promoción. Las viviendas con más de 30 años reflejan un $63 \%$ mientras que los inmuebles con menos de 30 años solamente representan un $37 \%$ del total (ver gráfica 3).

\section{Vivienda menor a 30 años}

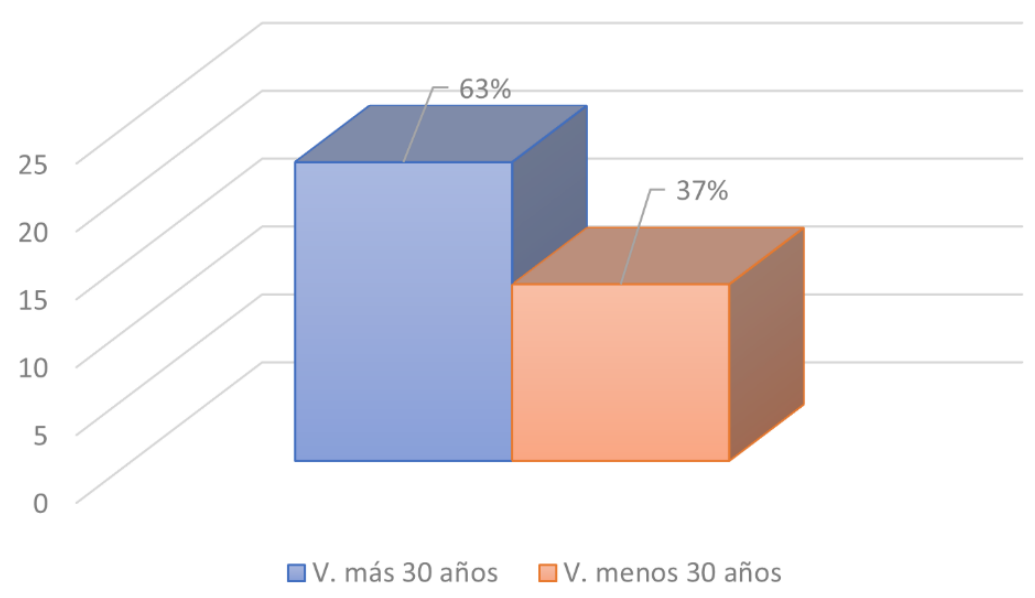

Grafica 3. Vivienda con edad menor a 30 años. Elaboración propia, 2021

En el estudio también se detectó la institución que genera mayor cantidad de créditos para la adquisición de vivienda. El INFONAVIT se visualiza con un $91 \%$ de créditos otorgados. Le siguen el FOVISSSTE, mercado abierto MAI y los créditos bancarios, estos últimos tres con solo un $3 \%$ cada uno. El INFONAVIT se establece con mayor determinación como la institución con mayor presencia en el otorgamiento de créditos hipotecarios (ver gráfica 4). 


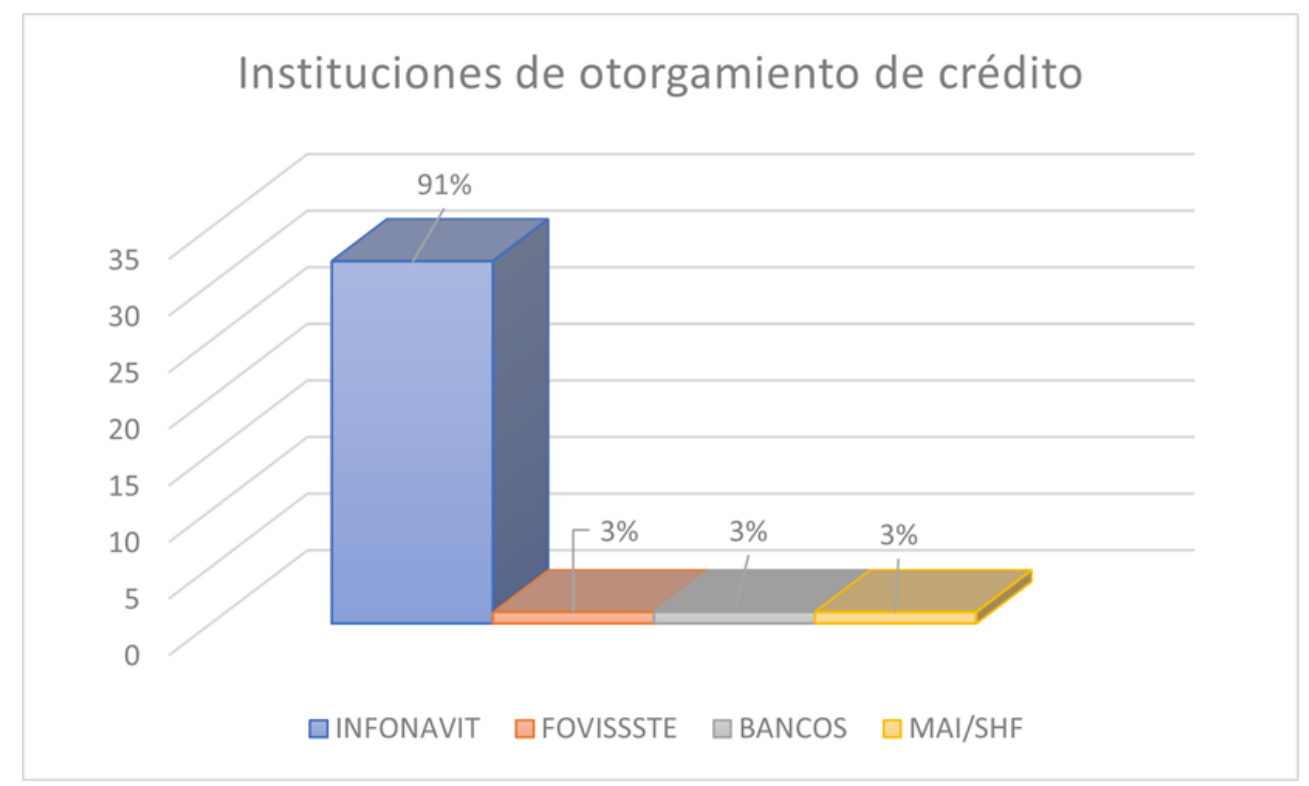

Gráfica 4. Instituciones de otorgamiento de crédito.

Elaboración propia, 2021.

En el presente estudio se identificó el tipo de vivienda donde se utiliza la tabla de prorrateo con mayor frecuencia. La vivienda de interés social se diagnóstica con un $91 \%$, siendo así el tipo de inmueble en donde se requiere este instrumento. La vivienda de interés medio aparece con solamente un $9 \%$, reflejando un porcentaje bajo con respecto a la vivienda de interés social. Por lo tanto, la vivienda de interés social se posiciona como el tipo de inmueble que maneja con mayor frecuencia esta herramienta con el objetivo de ser más comercial y volver a colocarse en el mercado como una opción de compra para diferentes tipos de personas que son susceptibles a un crédito hipotecario (ver gráfica5). 


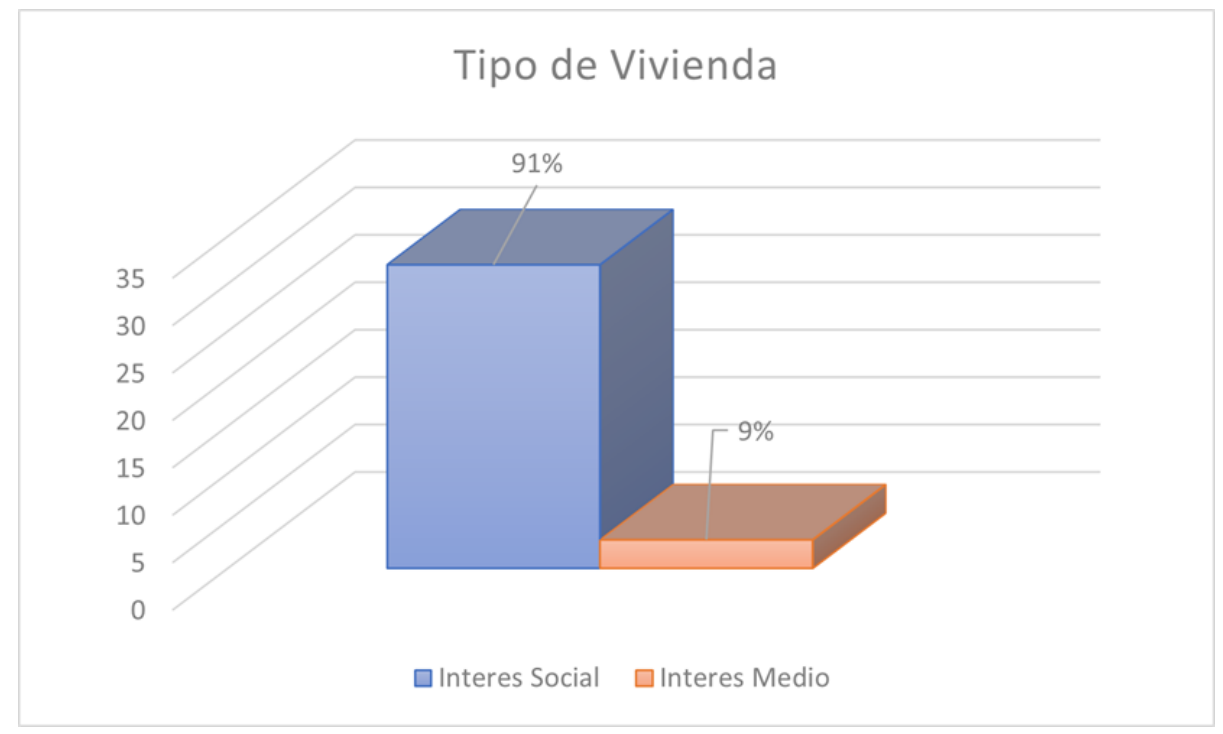

Gráfica 5. Tipo de vivienda que utiliza la tabla de prorrateo de edad.

Elaboración propia. 2021

En el campo de los valores de estas viviendas se obtuvo la siguiente información. Las viviendas con un precio por debajo de un millón de pesos representan el $86 \%$. Las viviendas con un costo arriba del millón de pesos aparecen solamente con un $14 \%$. Esta situación se traduce derivado que la mayor cantidad de viviendas que emplean este instrumento son viviendas o departamentos de interés social, a esto se relaciona con los porcentajes y la relación con el tipo de vivienda (ver gráfica 6).

\section{Valores Viviendas}

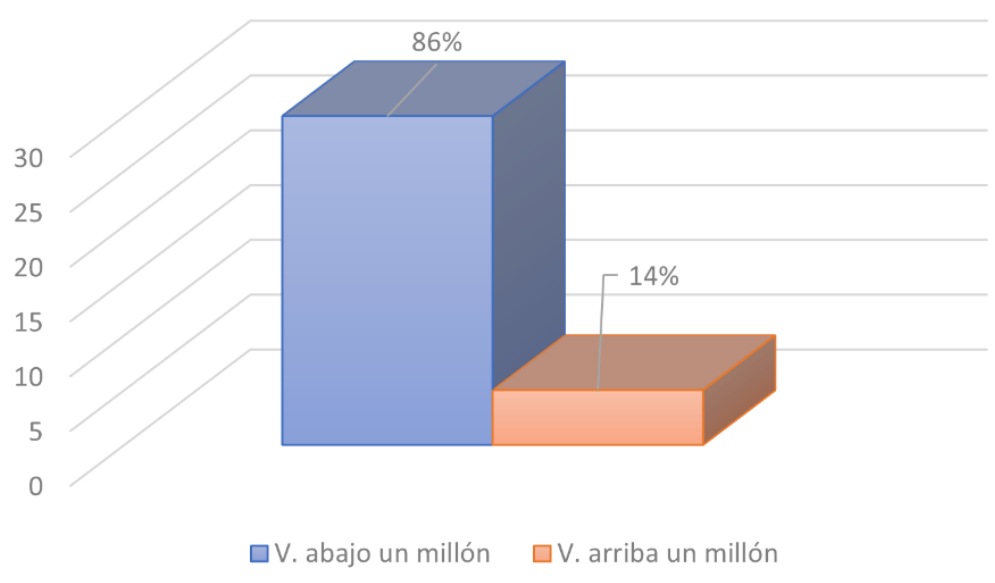

Gráfica 6. Porcentajes de viviendas con un valor arriba de un millón de pesos. Elaboración propia, 2021. 
En el renglón de la clasificación de vivienda las casas habitación representan un 63\%, mientras que los departamentos en régimen de condominio aparecen solamente con un 37\%. Esta información se analiza determinado que las casas habitación presentan mayor rango en aplicación de la tabla de prorrateo de edad, se requiere en mayor porcentaje este instrumento para lograr ubicar este inmueble como oferta de compra para derechohabientes (ver gráfica 7).

\section{Clasificación Vivienda}

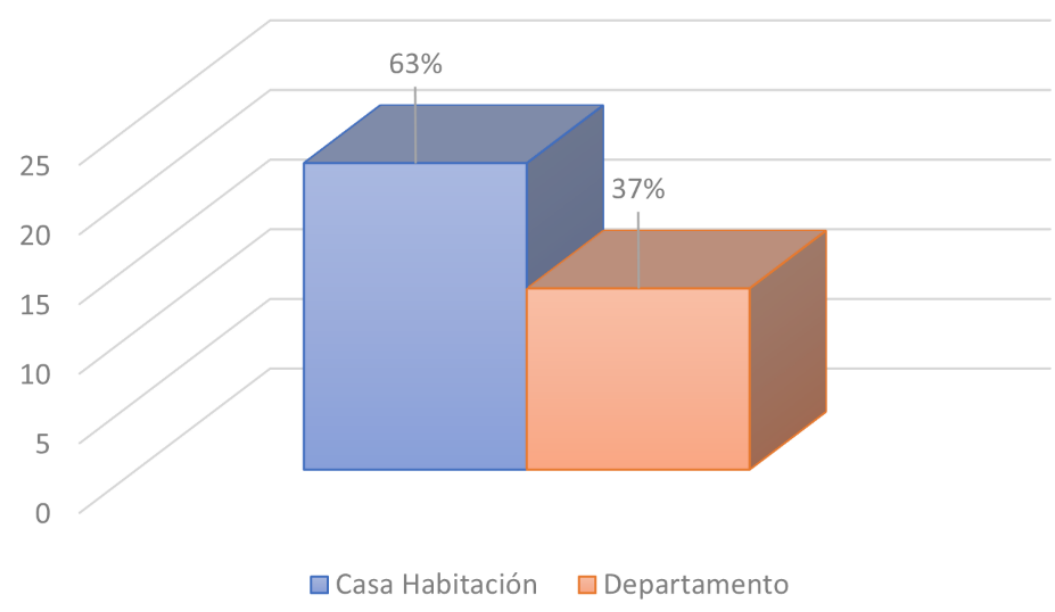

Gráfica 7. Clasificación de vivienda. Elaboración propia. 2021

En el diagnóstico realizado se logran conocer los municipios en donde se utilizó en mayor número esta herramienta. El Municipio de San Luis Potosí aparece con un $74 \%$, seguido del vecino municipio de Soledad de Graciano Sánchez con un 23\%, en un tercer plano con solamente un 3\% viviendas de otros municipios del interior del estado. Por lo tanto, el municipio de San Luis Potosí se ubica en primer lugar como zona para recurrir a este tipo de herramienta, derivado de la población que presenta este municipio se puede entender o relacionar el porcentaje que arrojo este estudio (ver gráfica 8). 


\section{Ubicación Municipio}

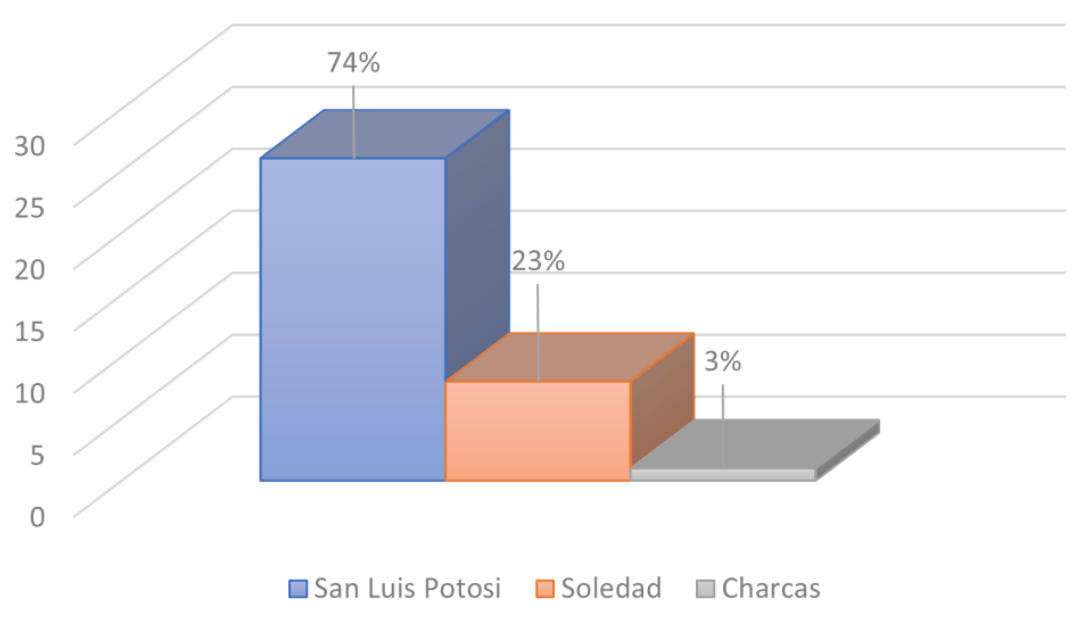

Gráfica 8. Localización de Municipios que utilizan la tabla de prorrateo. Elaboración propia. 2021

Finalmente, otro de los hallazgos de este estudio es que octubre y febrero son los meses cuando se utilizó más la tabla de prorrateo de edad. Esto se traduce como un mayor movimiento o solicitudes de créditos. En el mes de octubre se presenta esta situación ya que se visualiza el cierre del año y el sector inmobiliario se esfuerza en acomodar los últimos créditos. Para el caso del mes de febrero, se relaciona con el despegue del movimiento de créditos hipotecarios posterior a la cuesta de enero, momento que refleja el arranque del ejercicio de gestión de negocios de venta de inmuebles (ver gráfica 9). 


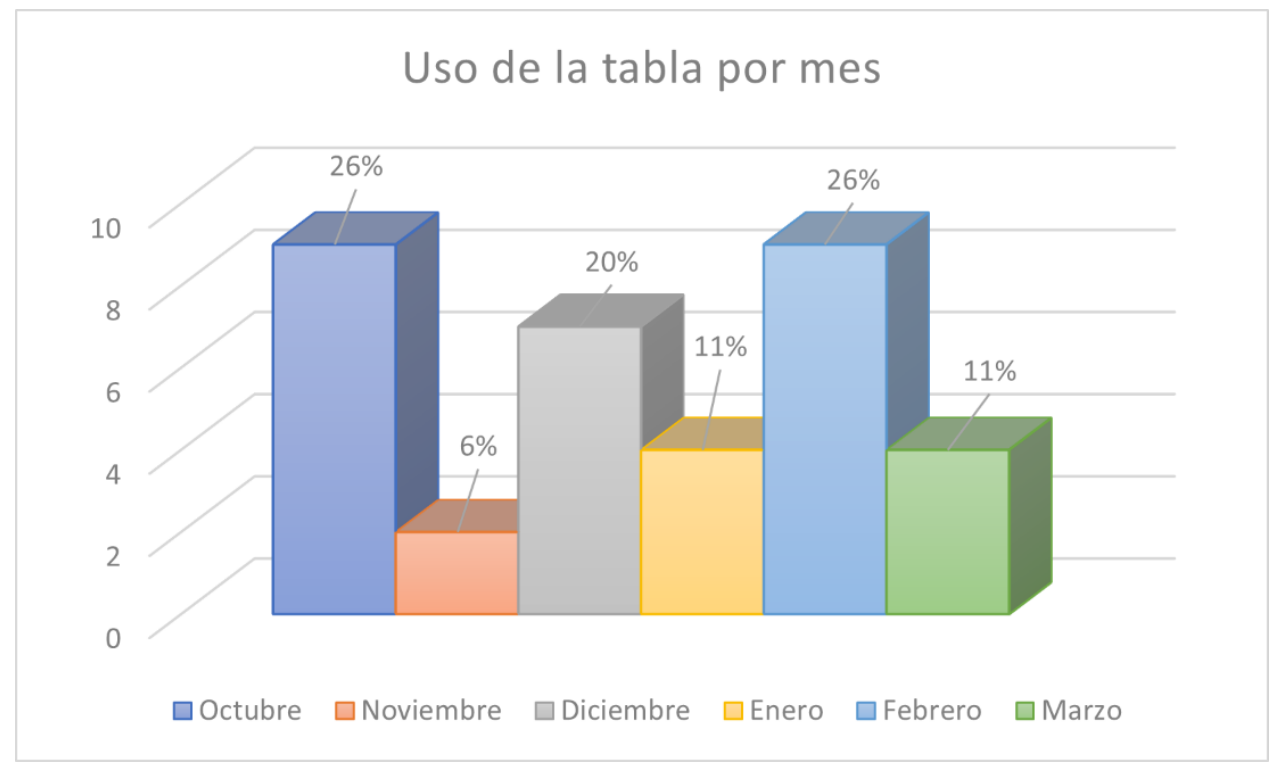

Gráfica 9. Uso de la tabla por mes.

Elaboración propia. 2021

\section{Conclusión}

Se puede determinar que esta nueva herramienta genera que una cantidad importante de viviendas que presentan una edad avanzada en la ZMSLP puedan ser nuevamente susceptibles a un crédito hipotecario con alguna institución privada bancaria o de gobierno. Por lo tanto, el objetivo de la presente investigación se alcanzó, generando áreas de oportunidad a compradores y vendedores del sector inmobiliario. Asimismo, coadyuva a desarrollar movimiento económico y ampliar las posibilidades de los jóvenes de adquirir una vivienda en zonas con inmuebles con edad avanzada.

Este estudio permite visualizar de una mejor manera las zonas y ubicación donde se utilizó la tabla de prorrateo, así como el tipo, el valor y la clasificación de vivienda en donde se empleó el instrumento, encontrando que la vivienda de interés social es hasta el momento el sector que recurre mayormente a esta herramienta.

El campo de la valuación aporta con este tipo de recursos, nuevas estrategias para el campo inmobiliario, reutilizando viviendas con una vida mayor a 30 años que son poco atractivas para futuros comparadores. Aunque posean una ubicación óptima, son candidatas como selección para jóvenes o persona adultas que utilizan un crédito hipotecario inclusive en una segunda ocasión.

La implementación de este tipo de herramientas genera trabajo para personas del campo de la construcción, gestores inmobiliarios, valuadores y vendedores de viviendas. Lo más importante de este estudio es motivar y generar la reactivación de zonas y/o complejos habitacionales donde sus 
unidades rentables presentan una mejor ubicación. Para lo cual solamente se requiere de planear adecuadamente una restauración estratégica de sus espacios y fomentar finalmente la reutilización de estas viviendas.

Son muchos los retos de la situación actual de la adquisición de vivienda en San Luis Potosí. En particular preocupan dos: i) las limitantes que impone el entorno económico para los jóvenes que buscan adquirir una vivienda propia; ii) la reutilización de casas que todavía se encuentran en buenas condiciones de habitabilidad y que por desconocimiento de este instrumento no se consideran una opción viable de compra. Por lo tanto, este estudio representa el primer esfuerzo por localizar todas las viviendas en la ZMSLP susceptibles a reincorporarse al mercado inmobiliario a través del uso de la metodología expuesta. De esta forma, se recomienda en una primera etapa realizar un censo detallado que contemple la edad, la ubicación y un presupuesto paramétrico de las mejoras de los inmuebles. Lo anterior, integraría una base de datos de suma importancia para difundir, en una segunda etapa, la información entre arquitectos y edificadores jóvenes que busquen un nicho de trabajo y a su vez colaboren para que las nuevas generaciones de la población económicamente activa adquieran un bien inmueble.

\section{References:}

1. Álvarez, F. (2012). Introducción a la Valuación de Inmuebles. Zacatecas, Zacatecas, México: Instituto Universitario de Posgrados en Alta Dirección.

2. Bellver, J. A. (2021). Valoración Inmobiliaria. Métodos y Alcances. Univsersitat Politécnica de Valéncia.

3. Consorcio Técnicos Inmobilairios, S. d. (10 de Octubre de 2020). Imagen 3. Tabla de prorratep de edad. Ciudad de México, Ciudad de México, México.

4. Cordova, J. (2017). Phoenix, valuación inmobiliaria (Vol. 1). Irapuato, Guanajuato, México: Phoenix.

5. Córdova, J. (2019). Valuación Inmobiliaria (Vol. 2a. edición). Irapuato, Guanajuato, México.

6. Diario Oficial. (14 de Septiembre de 2004). Reglas de Caracter General que Establecen la Metodología para la Valuación. Ciudad de México, Ciudad de México, México.

7. Garcia, M. (2007). Introducción a la Valoración Inmobilairia (Vol. 2a. edición). Cataluña: Universidad Politécnica de Cataluña.

8. Guerrero, O., Hernández, A., \& Pacheco, V. (2007). Undiades de Valuación, controladoras de riesgo. México: Centro Urbano. 
9. INFONAVIT. (21 de Abril de 1972). Instituto del Fondo Nacional de la Vivienda para los Trabajadores. Ciudad de México, Ciudad de México, México.

10. Mello, J. E. (20 de Septiembre de 2007). Diario Oficial de la Federación. Lineamientos Generales del Registro de Oferta de Vivienda para el FOVISSSTE. Ciudad de México, Ciudad de México, México.

11. Pérez, O. (2007). La agenda pendiente en la Valuación Profesional. Valuador Profesional, 6-9.

12. Salas, J. (2015). El Modelo de Valuación Inmobiliaria en México. Revista Iberoamericana para la investigación y el Desarrollo Educativo.

13. Salazar, C. S. (2013). Costo y tiempo en Edificación. Ciudad de México: Limusa.

14. SHF. (11 de Octubre de 2001). Sociedad Hipotecaria Federal. Ley Orgánica de la Sociedad Hipotecaria Federal. México.

15. Zhu, M. (5 de Junio de 2014). Los mercados inmobiliarios, la estabilidad financiera y la economía. China. 